\title{
Equilibrium Strategy and Population-Size Effects in Lowest Unique Bid Auctions
}

\author{
Simone Pigolotti, ${ }^{1,2}$ Sebastian Bernhardsson, ${ }^{1,3}$ Jeppe Juul, ${ }^{1}$ Gorm Galster, ${ }^{1}$ and Pierpaolo Vivo ${ }^{4}$ \\ ${ }^{1}$ Niels Bohr Institute, Blegdamsvej 17, DK-2100, Copenhagen, Denmark \\ ${ }^{2}$ Dept. de Fisica i Eng. Nuclear, Universitat Politecnica de Catalunya Edif. GAIA, \\ Rambla Sant Nebridi s/n, 08222 Terrassa, Barcelona, Spain \\ ${ }^{3}$ Swedish Defence Research Agency, SE-147 25 Tumba, Sweden \\ ${ }^{4}$ Univ. Paris-Sud, CNRS, LPTMS, UMR8626, Orsay F-01405, France
}

(Received 30 April 2011; published 22 February 2012)

\begin{abstract}
In lowest unique bid auctions, $N$ players bid for an item. The winner is whoever places the lowest bid, provided that it is also unique. We use a grand canonical approach to derive an analytical expression for the equilibrium distribution of strategies. We then study the properties of the solution as a function of the mean number of players, and compare them with a large data set of internet auctions. The theory agrees with the data with striking accuracy for small population-size $N$, while for larger $N$ a qualitatively different distribution is observed. We interpret this result as the emergence of two different regimes, one in which adaptation is feasible and one in which it is not. Our results question the actual possibility of a large population to adapt and find the optimal strategy when participating in a collective game.
\end{abstract}

DOI: 10.1103/PhysRevLett.108.088701

PACS numbers: 02.50.Le, 89.65.Gh, 89.75.Da

In recent years, statistical physics has provided powerful tools to study both equilibria [1,2] and dynamical properties [3-6] of games where the number of players is large. So far, most of the efforts in this field have been focused on games in which interactions among players are pairwise, the most notable and studied example being the prisoner's dilemma [3]. However, there are many examples of collective games where a unique winner is singled out from a large population. In such cases, comparing theory with empirical data is particularly challenging: As the number of players increases, the statistical description becomes more appropriate. However, the complexity of the equilibrium strategy may also increase, thus making it more difficult for real agents to infer it from available information [7].

Online auctions provide a fertile, yet scarcely investigated ground for exploring this problem [8-11]: The availability of large data sets provides a unique opportunity to study whether strategies of real players actually maximize their winning chances. Here, we focus on lowest unique bid auctions. This game is interesting for two reasons. First, it is sufficiently simple to allow for a comprehensive mathematical analysis $[12,13]$. Second, many detailed auction records are freely available online. The game is formulated as follows: $N$ players can bid for an item of value $V$. The bid must be a multiple of a minimum amount, so that one can effectively consider bid amounts as natural numbers. The winner (if any) is the player who places the lowest bid, which is unique, i.e., no other player bid on that amount. All players must pay a fee $c$ to take part in the auction; additionally, the winner has to pay the bid amount.

In this Letter, we derive an explicit analytic expression for the symmetric Nash equilibrium of the lowest unique bid auction and explore its dependence on the total number of players $N$. To achieve an explicit solution we assume that $N$ is not fixed, but fluctuates according to a Poissonian distribution, in analogy with the choice of the grand canonical ensemble in equilibrium statistical mechanics. We then compare the expression with a large data set [14] where players are informed in advance of the total number of allowed bids $N$. We find a remarkable change in the data as $N$ increases: In the low $N$ regime (fewer than $\approx 200$ players), the theory predicts very well the bid distribution. In this regime, the game is effectively a lottery, since winning chances are evenly spread on any number. Conversely, in the large $N$ regime, the data deviate from the theoretical solution and rather follow an exponential distribution. Here, players fail to adapt to the optimal strategy and the winning chances are highly dependent on the chosen number.

In a symmetric Nash equilibrium, all players adopt the same strategy, and no player can benefit from changing strategy unilaterally, i.e., should any player change strategy, his expected payoff would be equal or worse. Consider $N$ individuals playing according to the same strategy $\mathbf{p}=$ $\left(p_{1}, p_{2}, p_{3}, \ldots\right)$, where $p_{k}$ is the probability of bidding the number $k$. Then, the distribution of bids is multinomial:

$$
\mathcal{P}(\mathbf{n})=\frac{N !}{n_{1} ! n_{2} ! \ldots} p_{1}^{n_{1}} p_{2}^{n_{2}} \ldots,
$$

where $\mathbf{n}=\left(n_{1}, n_{2}, n_{3}, \ldots\right)$ are the number of bids placed on each number $k$. For convenience, we introduce the generating function:

$$
\mathcal{G}_{N}(\mathbf{x})=\sum_{\{\mathbf{n}\}} \mathcal{P}(\mathbf{n}) x_{1}^{n_{1}} x_{2}^{n_{2}}, \ldots,=(\mathbf{x} \cdot \mathbf{p})^{N} .
$$


We now assume that $N$ is not fixed, but fluctuates according to a Poissonian distribution of mean $\lambda$, leading to a grand canonical generating function:

$$
\tilde{G}_{\lambda}(\mathbf{x})=\sum_{N} \frac{\lambda^{N} e^{-\lambda}}{N !} G_{N}(\mathbf{x})=\exp [\lambda(\mathbf{p} \cdot \mathbf{x}-1)]
$$

i.e., the number of bids on each number is also Poisson distributed with mean $f_{k}=\lambda p_{k}$. By differentiating $\tilde{\mathcal{G}}$ with respect to $\mathbf{x}$, it is straightforward to compute the probabilities $w_{k}$ of $k$ being the winning number, i.e., that there is a unique bid on $k$ and no unique bids on lower numbers:

$$
w_{k}=f_{k} e^{-f_{k}} \prod_{j=1}^{k-1}\left(1-f_{j} e^{-f_{j}}\right) \text {. }
$$

It is also useful to compute the probability $c_{k}$ of $k$ being a potential winning number, i.e., that there are no bids on $k$ and no winners on lower numbers:

$$
c_{k}=e^{-f_{k}} \prod_{j=1}^{k-1}\left(1-f_{j} e^{-f_{j}}\right) .
$$

We note that the probability of each bid to be a winner on a given number $w_{k} / f_{k}$ is equal to the probability $c_{k}$ of numbers to be potential winning numbers, which is a peculiar property of Poisson games [15].

The expected payoff for a player bidding $k$ will be $(V-k) w_{k} / f_{k}-c$. At equilibrium, the expected payoff should be independent of $k$. Otherwise, players could benefit from bidding on numbers with high payoffs more frequently. Imposing this condition leads to a recurrence relation for the average bidding frequencies,

$$
f_{k+1}=\ln \left(e^{f_{k}}-f_{k}\right)+\ln \left(1-\frac{1}{V-k}\right) .
$$

To avoid a negative number of bids, the support of the distribution must be limited to the region where all $f_{k}$ 's are positive. In this region $k<V$ holds, so that the $f_{k}$ 's are strictly decreasing. The initial condition $f_{1}$ has to be determined iteratively from the condition $\sum_{j} f_{j}=\lambda$.

If the frequencies $f_{k}$ tend to zero for values of $k$ much smaller than the value of the item $V$ (a condition that is always fulfilled in our data set, and will be consistently assumed in the following), the last term in (6) can be disregarded. In this limit (formally corresponding to $V \rightarrow \infty)$ the recurrence relation has been derived in [13] and it is well defined for all values of $k \in \mathcal{N}$. We extend this solution by noting that the normalization condition $\sum_{k} f_{k}=\lambda$ implies an explicit expression of $f_{1}$ : Eq. (6) can be written as $f_{k}=e^{f_{k}}-e^{f_{k}+1}$, which summed over $k$ yields the initial condition $f_{1}=\ln (\lambda+1)$. This allows for an explicit expression for any specific $f_{k}$ 's by iteration. Substituting the solution into (4) also shows that the average chance of winning of each bid is equal to $(\lambda+1)^{-1}$, which is also equal to the chance of having no winner in the auction.

Before discussing the comparison with the data, we briefly study some properties of the Nash equilibrium distribution. When $f_{k} \gg 1$, a continuous approximation of (6) shows that $f_{k}$ decreases logarithmically with $k$. For small $f_{k} \ll 1$, one can approximate $f_{k+1} \approx f_{k}^{2} / 2$, showing that the distribution has a superexponential cutoff. The scaling of the value $k^{*}(\lambda)$, around which the cutoff occurs, can be estimated in the following way. By removing all the players that bid on $k=1$ we change the average number of players by the amount $f_{1}$ giving $\lambda_{\text {new }}=\lambda_{\text {old }}-\ln \left(\lambda_{\text {old }}+1\right)$. This is equivalent to shifting the whole distribution by one along the $k$ axis, resulting in $k_{\text {new }}^{*}=k_{\text {old }}^{*}-1$. This scaling transformation in the continuum limit becomes $d \lambda / d k^{*}=$ $\ln (\lambda+1)$, with the solution $k^{*}(\lambda)=\operatorname{li}(1+\lambda)+C$, where $\operatorname{li}(z)=\int_{0}^{z} d t / \ln t$ is the logarithmic integral and $C$ is a constant of $\mathcal{O}(1)$.

We now move to the comparison of the equilibrium solution with empirical data from Ref. [14]. The data set includes 724 online auctions from April 2007 to January 2011 with a variable number of bids ranging from $N=26$ to $N=4748$. The number of allowed bids in a particular auction is announced before bidding starts, and the auction closes when this number is reached. Each player is allowed a limited number of bids. The average number of bids per player in the full data set is only 2.47 and very weakly dependent on $N$. In the Supplemental Material [16], we demonstrate with agent based simulations that allowing a small number of multiple bids per player does not alter significantly the equilibrium strategy. For this reason, in the subsequent analysis, we will neglect the effect of multiple bids by the same player and treat the bids as statistically independent.

In Fig. 1 we compare the theoretical and empirical bidding frequencies in different auctions having different numbers of players and different item values. In order to make the histograms smoother, we averaged each panel over $L$ different auctions having similar numbers of players and same item value (shown in the figure).

On the fine scale of single numbers, the data show a structure dictated by known psychological effects. Players tend to favor odd numbers over even numbers. Some specific numbers (like 17 and other primes) are perceived to be "original" by some players, and are chosen with significantly larger probability than neighboring ones.

On a coarser scale, the agreement between theory and data is striking for smaller auctions [i.e., fewer than 200 players, panels (A) and (B) of Fig. 1]. It is particularly remarkable that the empirical histograms reproduce the sharp cutoff, considering the nontrivial dependence of $k^{*}$ on the number of players.

Theoretically, players should adjust their bidding strategies according to the number of players rather than the 

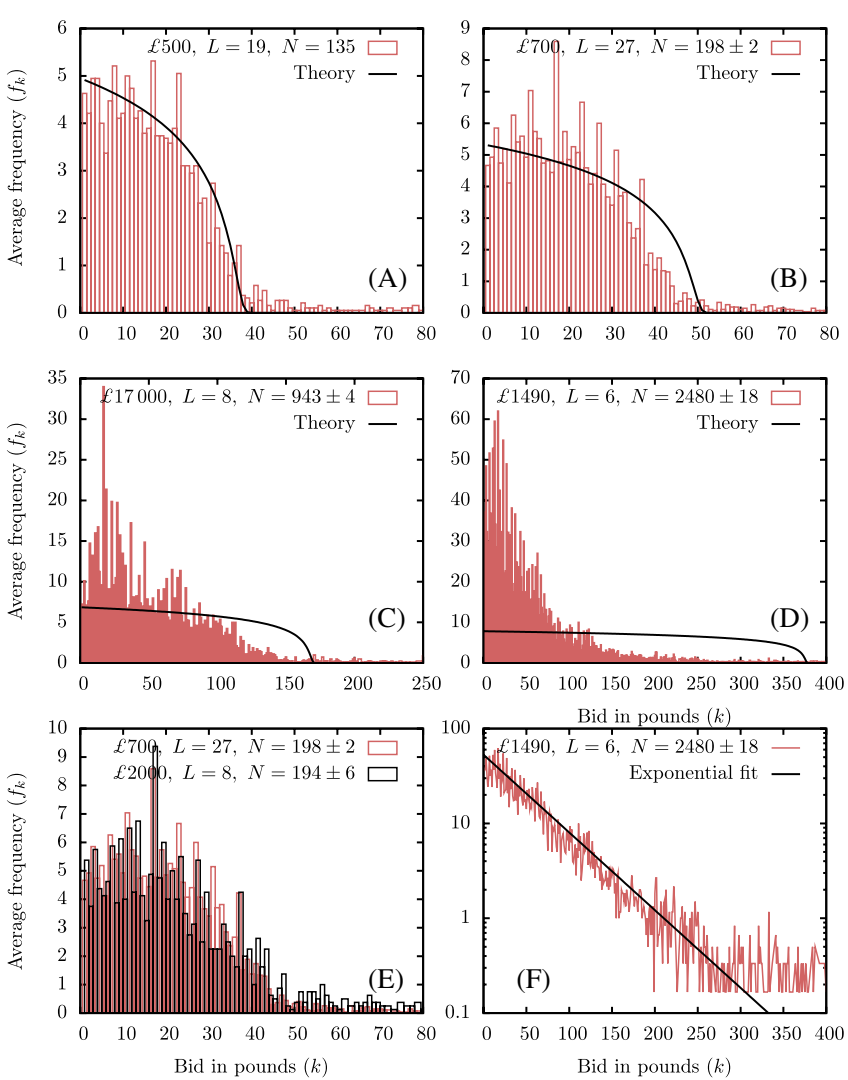

FIG. 1 (color online). Histograms of bidding frequencies compared with the theoretical equilibrium distribution. Panels (A)-(D) show average histograms of different auctions with a similar number of players and same item value. Panel (E) compares histograms of auctions with a similar number of players, but significantly different item values. Panel (F) is same as panel (D) but in linear-log scale, to illustrate the exponential shape of the empirical distribution at large $N$.

item value, which can be assumed to be infinite. In all auctions in the data set, the cutoffs of the corresponding theoretical distributions occur at bid values much smaller than the item values (shown in panels). To test whether the empirical bidding distributions are independent of $V$, panel (E) compares two sets of auctions with the same number of players, but with item values that differ by a factor of 3 . The bidding distribution for the pricey item have a slightly heavier tail, but overall the distributions are very similar.

The agreement between theory and data progressively deteriorates as the number of players increases [see panels (C) and (D)]. For a large number of players (more than 2000) an exponential distribution fits the data better, as shown in the lower panels of Fig. 1. This can be understood by arguing (see, e.g., [17-19]) that players having incomplete information about the game play according to a strategy defined as $p_{k}=\exp \left(-\beta E_{k}\right) / \sum_{j} \exp \left(-\beta E_{j}\right)$ where $E_{k}$ is the expected payoff when placing a bid $k$ and $\beta$ quantifies the degree of uncertainty about the game. If players have poor knowledge about the optimal

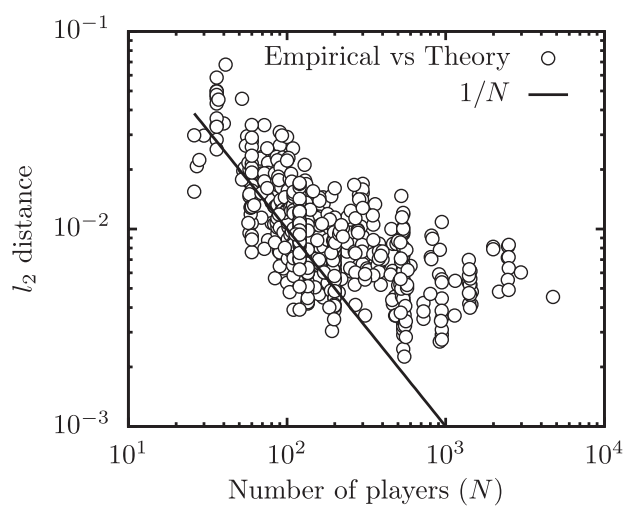

FIG. 2. $l_{2}$ distance between the theoretical solution and the empirical histogram for each auction. The continuous line is the theoretical expectation $\langle d\rangle=N^{-1}$ if all bids for each histogram were randomly drawn for the theoretical distribution.

strategy, they could assume a uniform prior probability to win on each $k$, resulting in $E_{k}$ decreasing linearly with $k$ due to the cost of the bid when winning. Substituting this prior into the aforementioned strategy yields a bid distribution exponentially decreasing with bid size.

A quantitative measure of how the data deviate from theory is shown in Fig. 2, where, for each auction, we plot the $l_{2}$ distance $d$ between the theoretical probability distribution and the empirical one, $d=N^{-2} \sum_{k}\left(f_{k}-\phi_{k}\right)^{2}$, where $\phi_{k}$ is the number of bids on $k$ in a given auction. If bids were randomly drawn from the theoretical distribution, the expected distance would decrease with the number of players as $\langle d\rangle=N^{-1}$. Empirically, the distances have a large spread around the expected value for small auctions and are consistently larger than expected for $N>200$. This outcome cannot be simply explained by the larger number of auctions for smaller $N$ : As a consequence of players' turnover, the distributions at fixed $N$ do not evolve with time in a significant way, as we checked by comparing older to more recent auctions.

Another interesting quantity is the distribution of actual winning numbers. At equilibrium, it should be equal to the distribution of bids. As shown in Fig. 3, the empirical distribution of winning numbers supports this feature. The vast majority of winning numbers fall within the theoretical cutoff (pink shaded area of Fig. 3). In the figure, we also show the analytical estimate $k^{*} \approx$ $(1+1 / \ln N) N / \ln N$ based on the asymptotic expansion of the logarithmic integral. The black line is the average winning number, which in the relevant range, scales approximately in the same way as the cutoff.

Binning the data yields average winning numbers in excellent agreement with the theory, even for large $N$ where the empirical distribution of bids departs from the theory. However, the variance of winning numbers becomes much smaller than the theoretical prediction for $N>10^{3}$. To further explore this phenomenon, we compute the actual probabilities to win on a certain number, given 

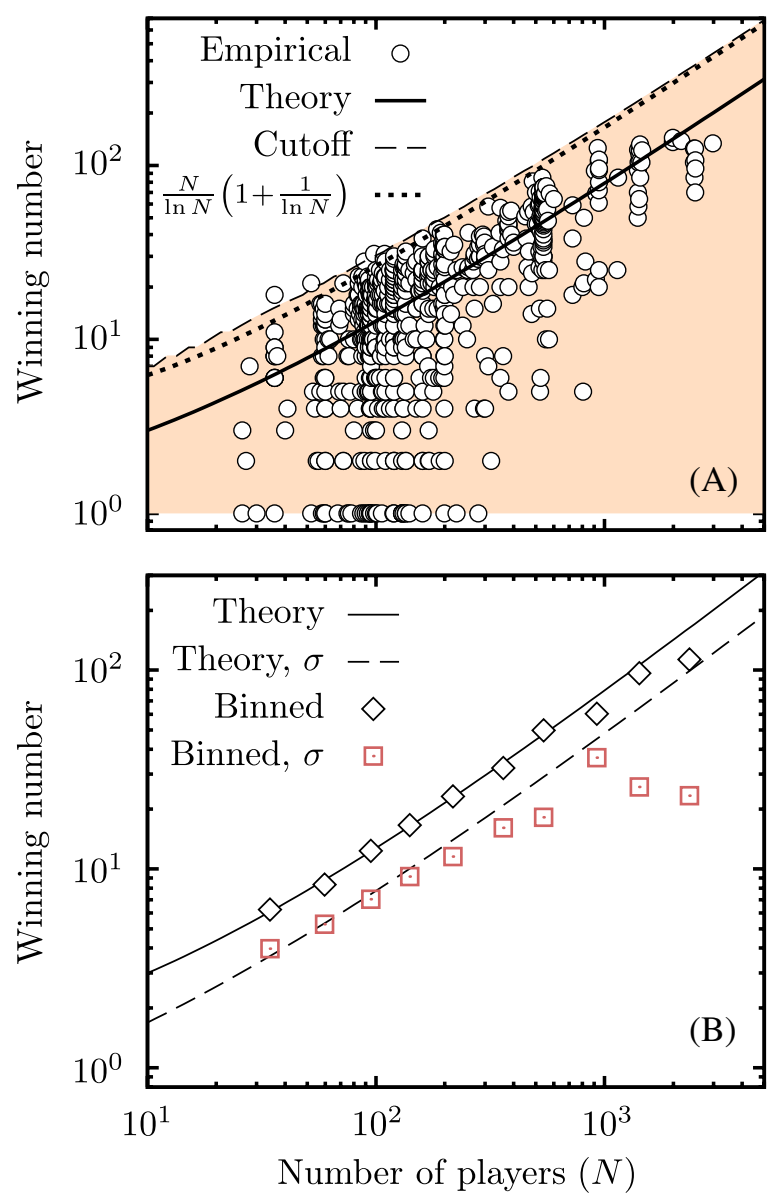

FIG. 3 (color online). Panel (A): Winning numbers as a function of auction size. The continuous line is the theoretical average and the shaded area denotes the region where bids are below the theoretical cutoff $k^{*}$. Panel (B): Average and standard deviation of the winning numbers, compared with the respective theoretical lines.

the empirical distributions of bids for auctions of various sizes. This probability is given by $w_{k} / f_{k}$ which, since $c_{k}=$ $w_{k} / f_{k}$, is the same as the probability to win on a certain number for an additional player entering the game. The results are shown in Fig. 4 for the same examples considered in Fig. 1. For auctions with few players, the largest chance of winning is not more than 4-5 times higher than $(N+1)^{-1}$, the winning probability at the Nash equilibrium. In this region, the game is not very different from a lottery, as the winning chances do not depend much on the chosen number $k \ll V$. In contrast, for large auctions, the winning chances on low and high numbers are very small, while intermediate bids may have a winning chance more than 60 times higher than the average bid in the Nash equilibrium, providing opportunities for exploitation and thus potential room for adaptation.

Summarizing, in this Letter we derived the analytical equilibrium bidding distribution for the lowest unique bid auction and compared it with empirical data. The emerging
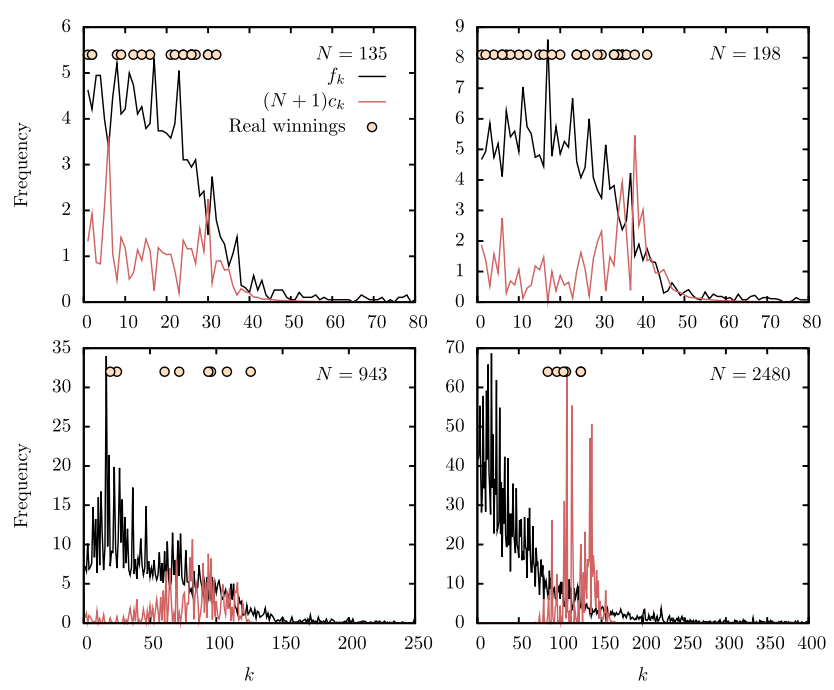

FIG. 4 (color online). Winning chances when bidding $k$. Black lines indicate the same empirical data as panels (A)-(D) of Fig. 1. Gray (red) lines are the relative probabilities to win when betting on $k$ given that all other players bid according to the empirical distribution. To help the comparison, we have renormalized the winning probabilities $c_{k}$ with their value for the Nash equilibrium $(N+1)^{-1}$. The shaded points mark the actual winning bids for each auction (arbitrary $y$ position).

picture is that players are able to infer the optimal strategy with striking accuracy when the number of players $N$ is not too large. In the large $N$ regime, the population-level strategy is highly nonoptimal and it seems to be determined by the simple principle of not assuming a particular preference for any number while avoiding the cost of large bids.

This result raises nontrivial questions about the effectiveness of adaptive dynamics in collective games. While it has been studied whether adaptation eventually drives the system to the optimal solution or generates more complex dynamical behaviors [20], it is also crucial to assess how fast the equilibrium is reached. A thorough study of adaptive dynamics in such a system will be the subject of a future study; in the Supplemental Material [16], we show that indeed, in the simple case of replicator dynamics, adaptation becomes slower at larger $N$. Such slowdown could prevent the inference of the equilibrium strategy for large populations in realistic time scales, and thus explain the increasing lack of adaptation in our data as $N$ grows.

We acknowledge useful discussions with M. Marsili, K. Sneppen, C. Strandkvist, and P. Kempson.

[1] D. Challet, M. Marsili, and R. Zecchina, Phys. Rev. Lett. 84, 1824 (2000).

[2] J. Berg and A. Engel, Phys. Rev. Lett. 81, 4999 (1998).

[3] C. Hauert and G. Szabó, Am. J. Phys. 73, 405 (2005).

[4] A. Traulsen, J.C. Claussen, and C. Hauert, Phys. Rev. Lett. 95, 238701 (2005). 
[5] A. Melbinger, J. Cremer, and E. Frey, Phys. Rev. Lett. 105, 178101 (2010).

[6] N. Hanaki, A. Kirman, and M. Marsili, J. Econ. Behav. Organ. 77, 382 (2011).

[7] S. K. Baek, P. Minnhagen, S. Bernhardsson, K. Choi, and B. J. Kim, Phys. Rev. E 80, 016111 (2009).

[8] A. Namazi and A. Schadschneider, Int. J. Mod. Phys. C 17, 1485 (2006).

[9] I. Yang, H. Jeong, B. Kahng, and A.-L. Barabási, Phys. Rev. E 68, 016102 (2003).

[10] J. Reichardt and S. Bornholdt, J. Stat. Mech. Theor. Exp. 2007, P06016 (2007).

[11] T. Galla, M. Leone, M. Marsili, M. Sellitto, M. Weigt, and R. Zecchina, Phys. Rev. Lett. 97, 128701 (2006).

[12] S. K. Baek and S. Bernhardsson, Fluctuation and Noise Letters 9, 61 (2010).
[13] R. Östling, J. T.-y. Wang, E. Y. Chou, and C. F. Camerer, American Economic Journal: Microeconomics, 3, 1 (2011).

[14] Available at http://www.auctionair.co.uk.

[15] R. B. Myerson, Int. J. Game Theory 27, 375 (1998).

[16] See Supplemental Material at http://link.aps.org/ supplemental/10.1103/PhysRevLett.108.088701 for additional data analysis and simulation results.

[17] R. D. McKelvey and T. R. Palfrey, Games Econ. Behav. 10, 6 (1995).

[18] H.-C. Chen, J. W. Friedman, and J.-F. Thisse, Games Econ. Behav. 18, 32 (1997).

[19] J. K. Goeree and C. A. Holt, Proc. Natl. Acad. Sci. U.S.A. 96, 10564 (1999).

[20] M. A. Nowak and K. Sigmund, Science 303, 793 (2004). 